- Original Article

\title{
Analysis of Clinical Manifestations and Laboratory Findings in Children with Influenza B-Associated Myositis: A Single Center Study
}

\author{
Jae Woong Yoon' ${ }^{1}$, Du Young Choi', Seung Hyun Lee', Sae Ron Sin², Seung Taek Yu',** \\ 'Department of Pediatrics, Wonkwang University School of Medicine, Iksan, Korea \\ ${ }^{2}$ Department of Family Medicine, Wonkwang University School of Medicine, Iksan, Korea
}

Background: Influenza-associated myositis (IAM) is a rare and poorly recognized complication of influenza infection in children, and is characterized by acute onset of severe pain in the lower extremities and a refusal to ambulate walk. We sought to understand the association between IAM and influenza B infection and to investigate its clinical and laboratory characteristics in affected children.

Methods: Influenza B-associated myositis (IBAM) cases diagnosed in the pediatrics department of Wonkwang University Hospital from January 2010 and March 2016 were analyzed retrospectively.

Results: Medical records of affected children were examined, and clinical characteristics and laboratory findings were recorded. Of the 536 children diagnosed with influenza B infection, 47 children complained of bilateral calf pain with or without gait disturbance. All children exhibited elevated serum aspartate aminotransferase (AST) level. The median serum creatine kinase (CK) and lactate dehydrogenase (LDH) levels, reportedly elevated in myositis, were 2,597 IU/L and $678 \mathrm{IU} / \mathrm{L}$, respectively. While the immunofluorescence test results were negative for some patients, the polymerase chain reaction test results indicated influenza B infection in all 47 children. At the time of hospital discharge, the patients' symptoms had resolved, and their CK levels had improved.

Conclusion: IBAM was generally benign and short, and although the blood AST, CK, and LDH levels were markedly high, the erythrocyte sedimentation rate and C-reactive protein levels were normal. Further, the duration of IBAM symptoms correlated with the duration of fever. The IBAM-associated clinical and laboratory findings are highly characteristic and may allow its rapid diagnosis during the influenza season.

Keywords: Child; Influenza B Virus; Myositis; Myalgia

Received: October 10, 2016, Revised: October 27, 2016, Accepted: October 28, 2016

${ }^{*}$ Corresponding Author: Seung Taek Yu https://orcid.org/0000-0001-9744-5548

Tel: +82-63-859-1510, Fax: +82-63-853-3670, E-mail: yudoc@wonkwang.ac.kr 


\section{INTRODUCTION}

Influenza is common and usually benign viral respiratory tract infection caused by the influenza virus, and annual influenza epidemics occur worldwide during the cold season. ${ }^{1)}$ The infections typically begin with predominantly systemic symptoms that include fever, chills, headache, myalgia, malaise, and anorexia. ${ }^{2,3)}$ Influenza is associated with a variety of complications ${ }^{4)}$ that primarily affect the respiratory tract and the central nervous system. Influenza-associated myositis (IAM), another complication of the infection, has only been reported sporadically since its first description in 1957. ${ }^{5)}$ Although IAM is more common in children than in adults, its age-specific incidence during influenza epidemics is unknown. IAM is a generally benign, self-limiting condition that usually affects boys and manifests as calf tenderness, and sudden onset of difficulty in walking after an influenza infection. ${ }^{6)}$ However, IAM may not be easily distinguishable from other diseases, and therefore, children may undergo unnecessary diagnostic testing and treatment. In addition, several IAM cases with a severe course and life-threatening complications have been also reported. ${ }^{\text {) }}$

IAM is typically associated with the Influenzavirus $B$, and previous reports demonstrate that influenza B infections cause myositis more often than influenza A infections. Although specific pathophysiology of IAM has not been established, the greater potency of Influenzavirus $B$ to directly invade muscle cells may result in greater muscle cell damage, and may be one explanation for the pathophysiological difference observed between influenza A and B infections. ${ }^{8)}$

Few previous studies have investigated IAM in childhood; however, the number of investigated cases was small in each of these studies. Further, no previous study has assessed the progression rate of myositis through an evaluation of laboratory findings and clinical symptoms like fever. Therefore, in our study, we aimed to assess the correlation between myositis progression and patients' laboratory findings and clinical symptoms. In addition, we studied the laboratory findings and clinical characteristics of influenza B-associated myositis patients in order to differentiate it from other severe myositis-inducing diseases and avoid unnecessary examinations.

\section{METHODS}

We retrospectively analyzed IBAM cases diagnosed in the pediatrics department of Wonkwang University Hospital from January 2010 to December 2015. The inclusion criteria for IBAM cases were as follows: (1) acute onset; (2) presence of calf muscle tenderness with or without a gait disturbance; (3) confirmed influenza B viral infection; (4) increased serum creatine kinase (CK) level; and (5) a self-limiting disease course. We performed chart reviews to collect data on clinical manifestations, age and sex of the patients, the duration of hospitalization, characteristics of influenza or influenza-like illness, muscular and other symptoms, laboratory findings (e.g., levels of hemoglobin [Hb], serum aspartate aminotransferase [AST], alanine aminotransferase [ALT], CK, lactate dehydrogenase [LDH], and C-reactive protein [CRP], the peripheral white blood cell [WBC], and platelet [PLT] counts, and the erythrocyte sedimentation rate [ESR]), urine analysis, duration and course of the illness, and the outcome of the illness.

\section{Microbiological Studies}

Flocked swabs and the universal transport medium (UTM-RT media; COPAN Diagnostics, Murrieta, CA, USA) were used to collect nasopharyngeal secretions from all subjects. Immunofluorescence assay (DFA Screening \& ID kit; Diagnostic Hybrid Inc., Athens, OH, USA) and multiplex polymerase chain reaction (PCR) tests (Seeplex TM RV detection kit; SeeGene Inc., Seoul, Korea) were used to detect influenza virus $\mathrm{B}$.

\section{Statistical Analysis}

We assessed each patient's clinical manifestations, laboratory findings and IBAM duration using Spearman's correlation test. Frequency analysis was conducted to estimate the mean, median, maximum, and minimum values for all laboratory results. All statistical analyses were performed with the help of PASW SPSS for Windows ver. 18.0 (SPSS Inc., Chicago, IL, USA). A $\mathrm{P}<0.05$ was considered a statistically significant.

\section{RESULTS}

Influenza infections are prevalent annually throughout Korea. During the period of influenza B epidemic between January 2010 and March 2016, 536 children were identified to have an influenza B infection based on PCR test results and the clinical manifestations of influenza.

Forty-seven children (8.7\%) complained of severe bilateral calf pain and exhibited noticeable gait disturbances. Other commonly manifested viral infection symptoms included fever, sore throat, cough, and coryza. Five children exhibited gastrointestinal symptoms, while neurological symptoms were not observed in any of the children (Table 1). During the same period, a total of 59 children complained of severe bilateral calf pain. Of them, 47 children tested positive for Influenzavirus $B$; four children tested positive for Adenovirus; and five children tested positive for Influenzavirus A, while no viral infection was detected in three children (Figure 1). The median age of 47 influenza B-infected children was 6.8 years. The median duration of fever was 3.8 days, the median duration from onset of fever to the onset of calf pain was 2.1 days, and the median duration from the onset of calf pain to the im-

Table 1. Clinical manifestation of influenza B-associated myositis in children

\begin{tabular}{lc}
\hline \multicolumn{1}{|c}{ Symptom } & No. of patients (\%) \\
\hline Fever & $47(100)$ \\
Calf pain & $47(100)$ \\
Cough & $40(85)$ \\
Coryza & $23(48)$ \\
Sore throat & $9(19)$ \\
Gait disturbance & $6(12)$ \\
Gastrointestinal symptoms & $5(10)$ \\
\hline
\end{tabular}


provement of calf pain was 4.2 days (Table 2 ).

The median WBC count was 5,194/ $\mathrm{mm}^{3}$ (range, 1,370-19,930/ $\mathrm{mm}^{3}$ ), and nine children had mild leukopenia. The Hb levels of all children were within the normal range. The median PLT count was $206 \times 10^{9} / \mathrm{L}$ (range, $60-491 \times 10^{9} / \mathrm{L}$ ), and eight children exhibited a mild thrombocytopenia. The median AST level was 152 IU/L (range, 66-433 $\mathrm{IU} / \mathrm{L}$ ), and the median ALT level was $50 \mathrm{IU} / \mathrm{L}$ (range, 15-329 IU/L). Median ESR was $8 \mathrm{~mm} / \mathrm{h}$ (range, $2-25 \mathrm{~mm} / \mathrm{h}$ ), and the median CRP level was $3.4 \mathrm{mg} / \mathrm{L}$ (range, 0.3-18.9 mg/L). The CK and LDH levels are typically elevated in myositis, and were also considerably high in our study population: 2,597 IU/L (range, 560-7,414 IU/L) and $678 \mathrm{IU} / \mathrm{L}$ (range, 367-1,232 IU/L), respectively. All urine analysis results were normal (Table 3 ). The duration of IAM symptoms did not correlate with the levels of $\mathrm{CK}(\mathrm{P}=0.101)$, serum AST $(\mathrm{P}=0.488)$, and serum ALT $(\mathrm{P}=0.489)$. However, the duration of IAM symptoms and the duration of fever demonstrated a significant correlation $(\mathrm{P}=0.001)$. Furthermore, even as some patients tested negative for Influenzavirus $B$ in the immunofluorescence test, all patients tested positive in the PCR test. None of the 47 children had any other influenza-related complications such as otitis media, pneumonia, myocarditis. At the time of discharge from the hospital, the children's presenting symptoms had resolved, and their CK levels had improved.

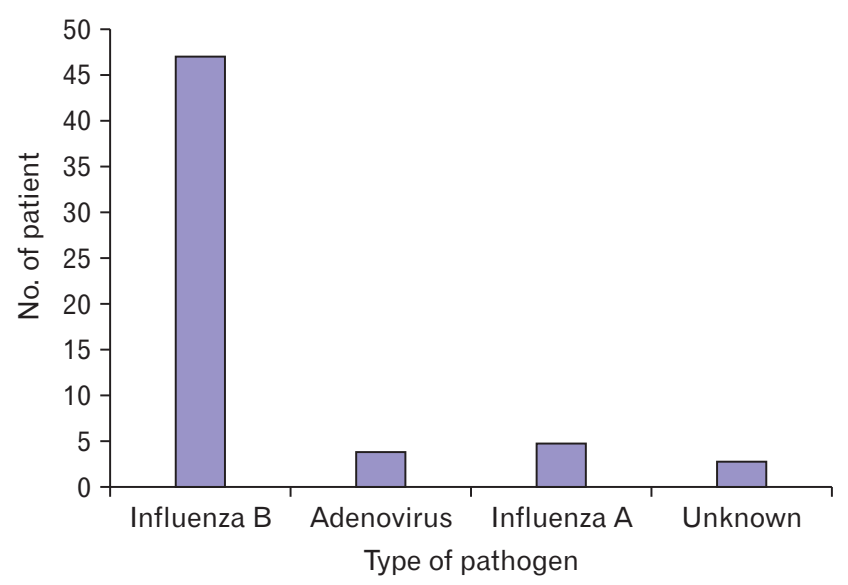

Figure 1. Number of children with calf pain classified by pathogens.

Table 2. Characteristics of influenza B-associated myositis patients

\begin{tabular}{lc}
\hline \multicolumn{1}{c}{ Variable } & \multicolumn{1}{c}{ Value } \\
\hline Age (y) & $6.8 \pm 3.1(2-18)$ \\
Sex (male/female) & $38 / 9(80.8 / 19.2)$ \\
Duration of hospitalization (d) & $5.7 \pm 2.3(3-17)$ \\
Fever duration (d) & $3.8 \pm 1.5(1-7)$ \\
Period between onset of fever and calf pain (d) & $2.1 \pm 1.5(0-6)$ \\
Period between onset and improvement of calf pain (d) & $4.2 \pm 1.3(1-7)$ \\
\hline
\end{tabular}

Values are mean \pm standard deviation (range) or number (\%).

\section{DISCUSSION}

The influenza infections predominant throughout Korea during the winter season cause highly contagious acute respiratory infections. ${ }^{1)}$ Fever is the most characteristic symptom associated with an influenza infection, although general symptoms such as a headache, myalgia, fatigue, and sore throat; and respiratory symptoms such as a cough, excessive sputum production, and coryza may also be associated with the infection. In addition, abdominal pain, nausea, and vomiting are also reported occasionally. Although most patients recover in approximately 1 week, death may occur in influenza-infected patients with chronic pulmonary diseases, heart diseases, or immunodeficiency-related diseases due to complications like pneumonia. Patients may also experience other complications such as encephalopathy, myelitis, Reye's syndrome, myositis, cardiomyositis, and pericarditis. ${ }^{1-5,8)}$

Symptoms of influenza complications linked to the musculature vary widely, from simple muscle pain to rhabdomyolysis. ${ }^{6,79)}$ IAM causes acute pain and muscular spasms during the recovery phase of the influenza infection, after the onset of respiratory symptoms. The IAM symptoms usually resolve within 3-10 days, and IAM thus has a good overall prognosis. ${ }^{10,11)}$

Benign acute childhood myositis (BACM) is primarily caused by an acute viral infection; the causative organisms include Influenzavirus $A$ and B, Parainfluenzavirus, Coxsackievirus, and Adenovirus, ${ }^{12)}$ among others. Mycoplasma infection-induced BACM has also been reported in a previous study. ${ }^{13)}$ In addition, Koliou et al ${ }^{14)}$ reported the case of an 11-year-old boy with myositis related to an infection with the new pandemic-causing Influenzavirus A (H1N1). However, severe muscle disease, particularly in children, is more frequently associated with an Influenzavirus B infection rather than those caused by other influenza viruses. ${ }^{10,15)}$

The first description of IAM as a distinct clinical entity dates back to 1957 when Lundberg ${ }^{5}$ reported 70 cases in children and 4 cases in adults of what he called "myalgia cruris epidemica". Middleton et al. ${ }^{15)}$ provided the first laboratory evidence supporting a link between influenza infection and IAM in the form of elevated CK and AST levels.

The pathophysiological mechanism of association between an Influenzavirus $B$ infection and IAM remains unknown; however, a possi-

Table 3. Laboratory findings in children with influenza B-associated myositis

\begin{tabular}{lc}
\hline \multicolumn{1}{c}{ Variable } & Value \\
\hline Hemoglobin $(\mathrm{g} / \mathrm{dL})$ & $12.3 \pm 0.9(10.5-14.3)$ \\
White blood cell $\left(/ \mathrm{mm}^{3}\right)$ & $5,194 \pm 3,329(1,370-19,930)$ \\
Platelet $\left(\times 10^{9} / \mathrm{L}\right)$ & $206 \pm 80(60-491)$ \\
Aspartate aminotransferase $(\mathrm{IU} / \mathrm{L})$ & $152 \pm 63(66-433)$ \\
Alanine aminotransferase $(\mathrm{IU} / \mathrm{L})$ & $50 \pm 49(15-329)$ \\
Creatine kinase $(\mathrm{IU} / \mathrm{L})$ & $2,597 \pm 1,530(560-7,414)$ \\
Lactate dehydrogenase $(\mathrm{IU} / \mathrm{L})$ & $678 \pm 160(367-1,232)$ \\
C-reactive protein $(\mathrm{mg} / \mathrm{L})$ & $3.4 \pm 4.5(0.3-18.9)$ \\
Erythrocyte sedimentation rate $(\mathrm{mm} / \mathrm{h})$ & $8.4 \pm 7.3(2.0-25.0)$ \\
\hline
\end{tabular}

Values are mean \pm standard deviation (range). 
ble explanation may be the greater direct muscle cell invading potential of the Influenzavirus B, that can, in turn, lead to greater muscle cell damage ${ }^{16)}$ In a small number of published pathological investigations, isolated segmental muscle fiber degeneration and necrosis without flanking myositis were noted, supporting the hypothesis of influenza virus-mediated direct muscle cell damage. ${ }^{17-20)}$

IBAM affects children and is predominately noted in boys aged 3-14 years. $^{21,22)}$ This male predominance may reflect a genetic predisposition or as yet unidentified metabolic defects. ${ }^{11,21)}$ The IBAM-associated myalgia appears abruptly, and may be severe, leading to gait disturbances or refusal to ambulate that may last for several days. Muscle swelling and tenderness may also occur in some cases.

In our study, the median age of IBAM onset among the 47 study subjects was 6.8 years, and the median duration from the onset of influenza symptoms to the onset of muscular symptoms was 2.1 days. In his study, Lundberg ${ }^{5)}$ reported the median age of onset as 9 years, while Mackay et al., ${ }^{21)}$ Bove et al., ${ }^{23)}$ and Agyeman et al. ${ }^{8)}$ reported median onset age as 8.1 years, 7 years, and 8.5 years, respectively. The median onset age in our study group was thus lower than that reported previously. The above-mentioned studies also reported the median duration between the onset of influenza symptoms and the onset of muscular symptoms as 2.6 days, 5 days, 5 days, and 3 days, respectively. In the 47 children in our study, and the IBAM-associated myalgia resolved after a median duration of 4.2 days, similar to the duration reported in the literature. ${ }^{5,8,21)}$

Our laboratory findings, including the significant increase in blood CK, AST, and LDH levels, matched previously published reports. ${ }^{21,22)}$ However, leukopenia and thrombocytopenia were not commonly noted in our study subjects; only 9 of the 47 children exhibited leukopenia, while eight children exhibited thrombocytopenia.

It is important to distinguish IBAM from more serious causes of gait disturbance and refusal to walk, such as influenza-associated myalgia, viral myositis associated with myoglobinuria, childhood idiopathic polymyositis, dermatomyositis, acute diffuse rhabdomyolysis, trichinosis, acute cerebellar ataxia, osteomyelitis, Guillain-Barré syndrome, deep vein thrombosis, juvenile rheumatoid arthritis, fracture, or malignancy. ${ }^{23-25)}$ Clinical findings not typically associated with IBAM include myoglobinuria, recent trauma or vigorous exercise, a family history of neuromuscular disorders, subacute or chronic progression, a new rash, any frank muscle weakness, or abnormal neurological findings. When the above-mentioned atypical features are present, disorders other than IBAM must be considered. ${ }^{26,27)}$ Dermatomyositis is characterized by a subtle onset, a chronic disease course, and skin involvement. Arthritis is frequently asymmetrical in distribution, and the associated CK values fall within the normal range. The Guillain-Barré syndrome is associated with absent tendon reflexes. Furthermore, deep venous thrombosis is exceedingly rare in otherwise healthy children, and usually involves only one extremity; it can be confirmed by a Doppler sonography and D-dimer level estimation in the blood. ${ }^{6}$ )

Furthermore, if the muscular pain, swelling, and tenderness worsen rapidly, or the condition does not resolve within a few days, additional urine analysis and further investigation of renal parameters are indicated. In cases where rhabdomyolysis is suspected, inpatient monitoring is indicated for early identification and treatment of associated complications such as acute renal failure, electrolyte imbalance, and compartment syndrome. ${ }^{6)}$

The use of imaging modalities, such as magnetic resonance imaging (MRI), has been described in the diagnosis of IBAM; however, an MRI may be more appropriate for the exclusion of other causes of myositis. ${ }^{28,29)}$

The hallmark of IBAM is its spontaneous and rapid clinical resolution within a week of symptom onset. ${ }^{21)}$ Treatment is supportive, and antiviral drugs are unlikely to be beneficial when started after the acute onset of viral infection; moreover, their benefit in cases of severe influenza, when administered after more than 2 days of symptom onset, is uncertain. $^{2)}$

The association between vaccination against influenza and the risk of developing IBAM is unknown. Our study did not assess the influenza vaccination history of the enrolled children. Although one may assume that influenza vaccine uptake may lower the risk of developing common post-influenza complications, a systematic review did not identify a decrease in secondary complications in vaccinated subjects. $^{30)}$

Although the clinical course of IBAM is very consistent, diagnosis is often not straightforward in the outpatient settings, and IBAM may be confused with other diseases. The etiology of IBAM is not entirely clear, and further laboratory investigations are not usually indicated in typical cases. Patients with IBAM should, therefore, be protected from unnecessary invasive procedures such as muscle biopsies, and specialized, expensive investigations such as brain MRI, electromusculogram, and nerve conduction studies. Further, the patients and their parents should be reassured of the generally benign disease course, and clinical, and laboratory follow-ups should be used to exclude serious diseases such as rhabdomyolysis.

This study has some limitations. We relied on a retrospective study design, with a small number of patients, in a specific area, at a single center. To assess the association between IBAM duration and laboratory findings, further multicenter studies with larger sample size are needed.

In conclusion, IBAM patients showed a benign disease course with abrupt symptom onset, usually affecting the lower extremities, and a short disease duration, in addition to the symptoms of influenza. These clinical characteristics may aid differential diagnosis in children with myalgia during influenza B epidemics and may help avoid unnecessary testing procedures. Although the blood AST, CK, and LDH levels were markedly increased in our study population, the ESR, and the CRP levels were normal. Moreover, even as the CK, AST, and ALT levels were not associated with the duration of IBAM symptoms, the duration of fever was associated with the duration of symptoms. Therefore, even though it may be difficult to predict disease progression on the basis of laboratory findings, the duration of fever may be considered predictive of the disease progression. 


\section{CONFLICT OF INTEREST}

No potential conflict of interest relevant to this article is reported.

\section{ACKNOWLEDGMENTS}

This work was supported by a research grant funded by Wonkwang University in 2018.

\section{REFERENCES}

1. Hu JJ, Kao CL, Lee PI, Chen CM, Lee CY, Lu CY, et al. Clinical features of influenza A and B in children and association with myositis. J Microbiol Immunol Infect 2004;37:95-8.

2. Fiore AE, Shay DK, Haber P, Iskander JK, Uyeki TM, Mootrey G, et al. Prevention and control of influenza: recommendations of the Advisory Committee on Immunization Practices (ACIP), 2007. MMWR Recomm Rep 2007;56(RR-6):1-54.

3. Stamboulian D, Bonvehi PE, Nacinovich FM, Cox N. Influenza. Infect Dis Clin North Am 2000;14:141-66.

4. Siberry GK. Complications of influenza infection in children. Pediatr Ann 2000;29:683-90.

5. Lundberg A. Myalgia cruris epidemica. Acta Paediatr 1957;46:18-31.

6. Mujgan Sonmez F, Cakir M, Yayla S, Boz C. Benign acute childhood myositis. Med Princ Pract 2004;13:227-9.

7. Cawkwell GM. Inflammatory myositis in children, including differential diagnosis. Curr Opin Rheumatol 2000;12:430-4.

8. Agyeman P, Duppenthaler A, Heininger U, Aebi C. Influenza-associated myositis in children. Infection 2004;32:199-203.

9. Christenson JC, San Joaquin VH. Influenza-associated rhabdomyolysis in a child. Pediatr Infect Dis J 1990;9:60-1.

10. Meier PW, Bianchetti MG. An 8-year-old boy with a 4-day history of fever, cough and malaise, and a 2-day history of painful calves and difficulty walking. Eur J Pediatr 2003;162:731-2.

11. Zafeiriou DI, Katzos G, Gombakis N, Kontopoulos EE, Tsantali C. Clinical features, laboratory findings and differential diagnosis of benign acute childhood myositis. Acta Paediatr 2000;89:1493-4.

12. Karpathios T, Kostaki M, Drakonaki S, Garoufi A, Siahanidou S, Spirou $\mathrm{N}$, et al. An epidemic with influenza B virus causing benign acute myositis in ten boys and two girls. Eur J Pediatr 1995;154:334-6.

13. Belardi C, Roberge R, Kelly M, Serbin S. Myalgia cruris epidemica (benign acute childhood myositis) associated with a Mycoplasma pneu- monia infection. Ann Emerg Med 1987;16:579-81.

14. Koliou M, Hadjiloizou S, Ourani S, Demosthenous A, Hadjidemetriou A. A case of benign acute childhood myositis associated with influenza A (H1N1) virus infection. Clin Microbiol Infect 2010;16:193-5.

15. Middleton PJ, Alexander RM, Szymanski MT. Severe myositis during recovery from influenza. Lancet 1970;2:533-5.

16. Davis LE, Kornfeld M. Experimental influenza B viral myositis. J Neurol Sci 2001;187:61-7.

17. Gamboa ET, Eastwood AB, Hays AP, Maxwell J, Penn AS. Isolation of influenza virus from muscle in myoglobinuric polymyositis. Neurology 1979;29:1323-35.

18. Greco TP, Askenase PW, Kashgarian M. Postviral myositis: myxoviruslike structures in affected muscle. Ann Intern Med 1977;86:193-4.

19. Kessler HA, Trenholme GM, Harris AA, Levin S. Acute myopathy associated with influenza $\mathrm{A} / \mathrm{Texas} / 1 / 77$ infection: isolation of virus from a muscle biopsy specimen. JAMA 1980;243:461-2.

20. Ozawa H, Noma S, Nonaka I. Myositis and rhabdomyolysis with influenza infection. Nihon Rinsho 2000;58:2276-81.

21. Mackay MT, Kornberg AJ, Shield LK, Dennett X. Benign acute childhood myositis: laboratory and clinical features. Neurology 1999;53:2127-31.

22. Shen CF, Huang SC, Wang SM, Wang JR, Liu CC. Decreased leukocytes and other characteristics of laboratory findings of influenza virus infections in children. J Microbiol Immunol Infect 2008;41:294-300.

23. Bove KE, Hilton PK, Partin J, Farrell MK. Morphology of acute myopathy associated with influenza B infection. Pediatr Pathol 1983;1:51-66.

24. Heiner JD, Ball VL. A child with benign acute childhood myositis after influenza. J Emerg Med 2010;39:316-9.

25. Jain S, Kolber MR. A stiff-legged gait: benign acute childhood myositis. CMAJ 2009;181:711-3.

26. Dalakas MC, Hohlfeld R. Polymyositis and dermatomyositis. Lancet 2003;362:971-82.

27. Emery AE. The muscular dystrophies. Lancet 2002;359:687-95.

28. Kawarai T, Nishimura H, Taniguchi K, Saji N, Shimizu H, Tadano M, et al. Magnetic resonance imaging of biceps femoris muscles in benign acute childhood myositis. Arch Neurol 2007;64:1200-1.

29. Panghaal V, Ortiz-Romero S, Lovinsky S, Levin TL. Benign acute childhood myositis: an unusual cause of calf pain. Pediatr Radiol 2008;38:703-5.

30. Jefferson T, Smith S, Demicheli V, Harnden A, Rivetti A, Di Pietrantonj C. Assessment of the efficacy and effectiveness of influenza vaccines in healthy children: systematic review. Lancet 2005;365:773-80. 